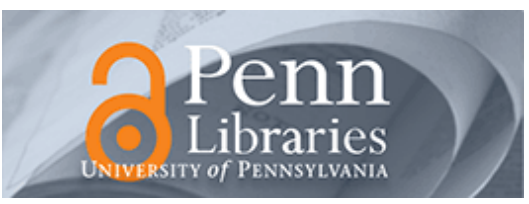

University of Pennsylvania

ScholarlyCommons

\title{
Optical Realization of the Retinal Ganglion Receptive Fields in Electron-Trapping Material Thin Film
}

\author{
Ramin Pashaie \\ University of Pennsylvania, raminp@seas.upenn.edu \\ Nabil H. Farhat \\ University of Pennsylvania, farhat@seas.upenn.edu
}

Follow this and additional works at: https://repository.upenn.edu/ese_papers

Part of the Electrical and Computer Engineering Commons

\section{Recommended Citation}

Ramin Pashaie and Nabil H. Farhat, "Optical Realization of the Retinal Ganglion Receptive Fields in Electron-Trapping Material Thin Film", . April 2006.

Suggested Citation:

Pashaie, R. and N. Farhat. (2006). "Optical Realization of the Retinal Ganglion Receptive Fields in Electron-Trapping Material Thin Film." Proceedings of the IEEE 32nd Annual Northeast Bioengineering Conference, 2006. April 1-2, 2006.

C2006 IEEE. Personal use of this material is permitted. However, permission to reprint/republish this material for advertising or promotional purposes or for creating new collective works for resale or redistribution to servers or lists, or to reuse any copyrighted component of this work in other works must be obtained from the IEEE.

This paper is posted at ScholarlyCommons. https://repository.upenn.edu/ese_papers/556

For more information, please contact repository@pobox.upenn.edu. 


\title{
Optical Realization of the Retinal Ganglion Receptive Fields in Electron-Trapping Material Thin Film
}

\author{
Abstract \\ Optical control of the electron-trapping material is used to model the retinal ganglion cell's receptive field. \\ Using this approach all the retinal image processing can be done on the surface of a thin film of this \\ material. \\ Disciplines \\ Electrical and Computer Engineering | Engineering \\ Comments \\ Suggested Citation: \\ Pashaie, R. and N. Farhat. (2006). "Optical Realization of the Retinal Ganglion Receptive Fields in Electron- \\ Trapping Material Thin Film." Proceedings of the IEEE 32nd Annual Northeast Bioengineering Conference, \\ 2006. April 1-2, 2006. \\ (C2006 IEEE. Personal use of this material is permitted. However, permission to reprint/republish this \\ material for advertising or promotional purposes or for creating new collective works for resale or \\ redistribution to servers or lists, or to reuse any copyrighted component of this work in other works must \\ be obtained from the IEEE.
}




\title{
Optical Realization of the Retinal Ganglion Receptive Fields in Electron-Trapping Material Thin Film
}

\author{
Ramin Pashaie, Nabil Farhat \\ University of Pennsylvania, Electrical and Systems Engineering Department, PA, 19104-6391
}

\begin{abstract}
Optical control of the electron-trapping material is used to model the retinal ganglion cell's receptive field. Using this approach all the retinal image processing can be done on the surface of a thin film of this material.
\end{abstract}

\section{INTRODUCTION}

VLSI (particularly CMOS technology) and optics are the two major approaches widely used for the implementation of the artificial retina [1-4]; however, optical realization of the parallel processing systems such as neural nets is superior to their electronic counterparts. In the present paper it's shown that the dynamics of the equilibrium-state emission of the electron-trapping material (ETM) under simultaneous blue light and near infrared (NIR) illumination can be designed to exhibit both excitatory and inhibitory response to the same optical stimuli. Because of this property, ETM can be considered as a potential candidate for the optical implementation of the concentric center-surround receptive field organization of the retinal ganglion cells.

\section{RETINAL INFORMATION PROCESSING}

Ganglion cells are the only cells in the laminar organization of the retina which project axons through the optic nerve. These type of cells recieve visual information from rod and cone photoreceptors. The region of space where photoreceptor outputs stimulate a ganglion cell is called the receptive field of the ganglion cell. Receptive fields of the ganglion cells are organized in a way that makes them capable of detecting contrast, edges and spatial frequency of the image. These receptive fields are arranged as two concentric regions, similar to the ones depicted in Figure 1, where the central disk is known as center and the circumscribed ring is called surround. Reaction of the ganglion cell's membrane potential to the light in the receptive field center is opposite to that of light in the surround. Based on the response of the ganglion cells to the center and surround regions, two distincted types of ganglion cells exsits, (on-center, off-surround) or (offcenter, on-surround). In the (on-center, off-surround), illumination of the center causes depolarization of the ganglion cell which increases its rate of firing; whereas, illumination of the surround will cause antagonistic hyperpolarization of the ganglion cell and reduction in the rate of firing. (off-center, on-surround) ganglion cells respond oppositely to the optical stimulation of the center and surround parts of their receptive fields. This contradictory reaction stems from the type of the neurotransmitters (amino acid glutamate), photoreceptors release in response to the optical stimulation that can cause both inhibitory and excitatory response in the ganglion cells, depending on their traget ion channels. Firing rate of the cell in the center compare to the one in the surround, encodes the contrast information of the optical stimulation and spatial frequency of the image can be extracted from the firing rate of the ganglion cells of different receptive field size. Small receptive fields are sensitive to high spatial frequencies and fine detail of image whereas larger receptive fields are sensitive to the low spetial frequencies. Center-surround organization can also encode information about discontinuities of the light distribution in the image which can be used for edge detection. Most of the retinal ganglion cell can be divided to one of the two categories, Magno-ganglion cells or Parvo-ganglion cells whose response properties differ in several ways. Magnoganglion cells, compare to Parvo-ganglion cells, have larger receptive fields and higher sensitivity to a low-contrast stimuli [5].

\section{DYNAMICS OF THE ELECTRON-TRAPPING MATERIAL}

Electron Trapping Materials (ETM) are alkaline-earth sulfides doped with rare-earth luminescence centers [6]. These rare earth doped elements add trap energy level within the host band-gap. Illumination of the ETM with blue light with peak wavelength around $450 \mathrm{~nm}$ excites some of the electrons of the valance band. Part of the excited electrons tunnel to the trap energy level and become trapped-electrons. Exposing the ETM to near infrared (NIR) light with peak wavelength around $1310 \mathrm{~nm}$ de-traps some of the trapped-electrons. Detrapped electrons return to the ground state by releasing their extra energy as an orange light emission with peak wavelength around $650 \mathrm{~nm}$. Under simultaneous blue light and NIR illumination, after a short transient period, the intensity of the orange light emission converges to a constant value, an equilibrium-state emission of the ETM. The intensity of the equilibrium-state emission of the ETM depends on the intensities of the blue light and NIR illumination and on parameters of the material. Extending the analysis of the dynamics of ETMs, given in $[7,8]$, we were able to show that the equilibrium-state emission of the ETM can be formulated by these two equations [9]:

$$
\begin{aligned}
& \frac{4 A}{B} I_{B} \operatorname{Sinh}^{2}\left(\frac{n_{s}-n}{2 A I_{B}}\right)=\frac{4 A^{\prime}}{B^{\prime}} I_{N I R} \operatorname{Sinh}^{2}\left(\frac{n}{2 A^{\prime} I_{N I R}}\right) \\
& I_{O}(t)=\alpha n(t) I_{B}+\beta n(t) I_{N I R}
\end{aligned}
$$




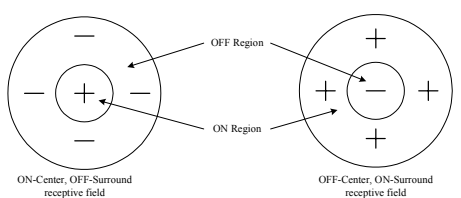

Fig. 1. Receptive fields of two different types of the ganglion cells.

In these equations $I_{B}, I_{N I R}$, and $I_{O}$ are the intensities of blue light, NIR and orange light emission respectively. $A, B, A^{\prime}, B^{\prime}$, $\alpha$ and $\beta$ are parameters of the material which are functions of wavelength. $n(t)$ represents the density of the trappedelectrons and $n_{s}$ is the saturation level of $n(t)$. Using these two equations, the intensity of the orange light emission for any specified values of the blue light and NIR intensities can be calculated. Figure 2 displays equilibrium-state plane of the ETM in which contours of constant orange light emission intensity are plotted as function of the intensities of the blue light and NIR exposures. Let's assume that the intensity of one of the light sources, say the NIR source, is a linear function of the intensity of the other source. This means that on the equilibrium state plane only orange light emission along a line segment are accessible. Few sample line segments are displayed in Figure 2. One subtle point that should be considered in the study of this diagram is that by changing the intensity of the blue light, the intensity of the orange light emission increases along $S \rightarrow 1$ line and decreases along $S \rightarrow 2$ line. As a result, the response of the ETM to the linearly coupled light sources along $S \rightarrow 1$ line is excitatory and along the line $S \rightarrow 2$ is inhibitory. By changing the slope of the $S \rightarrow 1$ and $S \rightarrow 2$ lines, the degree of excitation and inhibition of the ETM can be changed. In fact, by choosing right slopes one can model both Magno and Parvo ganglion cells.

\section{EXPERIMENTAL SETUP}

A schematic of the optical arrangement that can be used for the realization of an (on-center, off-surround) retinal ganglion receptive field is shown in Figure 3. Two pairs of linearly coupled light sources (B1, NIR1) and (B2, NIR2) are employed, where the first pair, whose light passes through the mask M1,

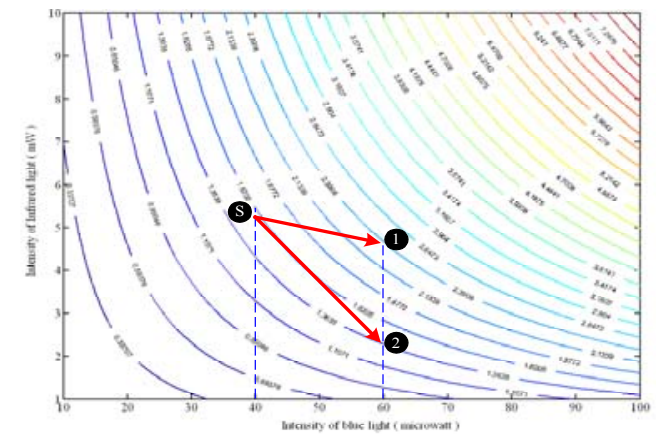

Fig. 2. Equilibrium-state emission plane of the ETM. Along the $S \rightarrow 1$ line the ETM is excitatory while its behavior along $S \rightarrow 2$ is inhibitory.

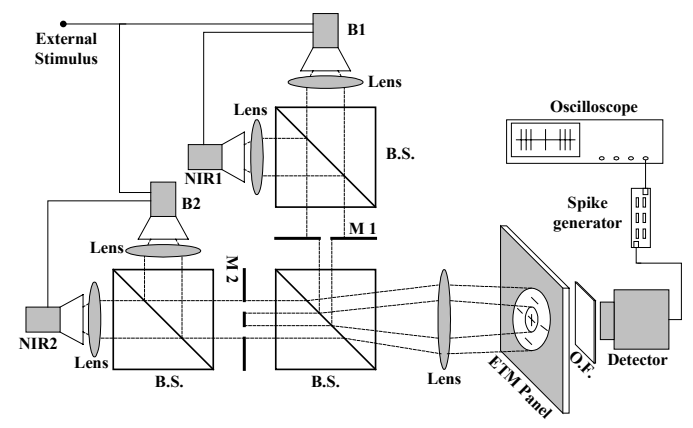

Fig. 3. Schematic of the setup.

illuminates the central region and the second couple, whose light passes through the mask M2, illuminates the surround area. (B1, NIR1) are allowed to take values in the dynamic range of $S \rightarrow 1$ line, which exhibits excitatory behavior unlike the (B2, NIR2), that are locked along $S \rightarrow 2$ line to behave inhibitory. A photo-detector, feeding a voltage controlled spike generator circuit, detects the orange light emission of the ETM through an orange optical filter (O.F.). The spikegenerator generates spikes, analogous to the membrane action potential, with spike rate that is function of the photodetector's output voltage level. Without any external stimulation setup is biased on point $S$ in the diagram. External stimulus is applied by increasing the intensity of the blue light, Which means that equilibrium-state orange light emission takes values along $S \rightarrow 1$ or $S \rightarrow 2$. Using this methodology an (on-center, off-surround) receptive field can be implemented on a thin film of ETM. By using appropriate masks, many receptive fields, with different sizes, can be produced on a thin film of ETM; however, this setup will become much more interesting when one replaces these two static masks and photo-detector by programmable spatial light modulators and CCD camera. Using this approach variety of retinal information processing can be done in ETM thin film.

\section{REFERENCES}

[1] H. Takei, A. Lewis, Z. Chen, I. Nebenzahl, "Implementing receptive fields with excitatory and inhibitory optoelectrical responses of bacteriorhodopsin films," Appl. Opt. 30 500-509 (1991).

[2] C. Wang, B. Jenkins, J. Wang, "Visual cortex operations and their Implementation using the incoherent optical neuron model," Appl. Opt. 32 1876-1887 (1993).

[3] K. Shimonomura, T. Yagi, "A multichip VLSI system emulating orientation selectivity of primary visual cortical cells," IEEE Trans. Neural Networks, Vol. 16, pp. 972-979, Jul. 2005.

[4] S. Kameda, T. Yagi, "An analog silicon retina with multichip configuration," IEEE Trans. Neural Networks, Vol. 17, pp. 197-210, Jan 2006.

[5] M. Bear, B. Connors, M. Paradiso, Neuroscience Exploring The Brain, Lippincott Williams \& Wilkins, $2^{\text {nd }}, 2001$.

[6] J. Lindmayer,' A new erasable optical memory'. Solid state technology, 1988.

[7] Z. Wen, N. Farhat, "Dynamics of electron trapping materials for use in optoelectronic neuro-computing," Appl. Opt. 32 7251-7265 (1993).

[8] Z. Wen, N. Farhat, "Electron trapping materials and electron-beamaddressed electron-trapping material devices: an improved model," Appl. opt. 34 5188-5198 (1995).

[9] R. Pashaie, N. Farhat, "Equilibrium-state emission of Electron-trapping materials and its applications", unpublished. 